\title{
Hawking Radiation via Tunneling from hot NUT-Kerr-Newman-Kasuya Spacetime
}

\author{
M. Hossain Ali 12
}

\begin{abstract}
We study the Hawking thermal spectrum in dragging coordinate system and the tunneling radiation characteristics of hot NUT-Kerr-Newman-Kasuya spacetime. The tunneling rates at the event and cosmological horizon are found to be related to the change of Bekenstein-Hawking entropy. The radiation spectrum is not pure thermal and thus there is a correction to the Hawking thermal spectrum.
\end{abstract}

PACS: 04.70.-s, 97.60.Lf

Keywords: Hawking radiation, conservation of energy and angular momentum, self-gravitation, horizons, tunneling rate, Bekenstein-Hawking entropy.

\footnotetext{
${ }^{1}$ Department of Applied Mathematics, Rajshahi University, Rajshahi-6205, Bangladesh.

${ }^{2}$ The Abdus Salam International Centre for Theoretical Physics, Strada Costiera 11, 34014 Trieste, Italy. E-mail: m_hossain_ali_bd@yahoo.com
} 


\section{Introduction}

The signifying discovery of Stephen Hawking [1, 2, 3] that black hole radiates thermally raised a disturbing and difficult problem, regarding information during black hole evaporation, called as the information loss paradox. Hawking's result also implies the loss of unitarity, or even the breakdown of quantum mechanics [4, that is, the pure quantum state is disintegrated to the mixture. In the language of Quantum Field Theory, the ingoing state is the pure state, but the outgoing is the mixture. Besides, Hawking regarded that the black hole radiation is created by pair of particles via tunneling from the black hole horizon as a result of the vacuum fluctuation. So there does exist a tunneling process, but the created mechanism of the tunneling barrier is unclear in this theory. The related references do not use the language of quantum tunneling method to discuss Hawking radiation, and hence it is not the quantum tunneling method. In order to derive the radiant spectrum from the black hole horizon, one must solve the two difficulties: first, the formed mechanism of the potential hill; and secondly, the elimination of the coordinate singularity.

Recently, Hawking changed his opinion regarding the information loss paradox and argued that information can indeed get out of the black hole [5]. Hawking's this argument is partly based on the Parikh-Wilczek's recent work [6, 7, 8] that treats the Hawking radiation as tunneling process and applies the semi-classical method to present the actual radiation as not exactly thermal but subtle correction to the Hawking thermal spectrum.

Parikh-Wilczek, combined with the above reasons, proposed a semi-classical quantum tunneling model that implemented Hawking radiation as a tunneling process. This model was actually initiated by Kraus and Wilczek ([9], [10]) and Keski-Vakkuri and Kraus [11], and was developed by Parikh and Wilczek with considerable success by carrying out a dynamical treatment of black hole geometry. More specially, they took into account the effects of a positive energy matter shell propagating outwards through the horizon of the Schwarzschild and Reissner-Nordström black holes, and incorporated the self-gravitation correction of the radiation ([7], [8], [12]). In this theory, the self-gravitation action among the particles creates the tunneling barrier with turning points at the location of the black hole horizon before and after the particle with energy emission. The derived result implies that the radiant spectrum is not thermal, but is consistent with the underlying unitary theorem. This framework is so successful that it also satisfies the first law of black hole thermodynamic. Following this method, the radiation from spherically symmetric AdS black hole [13] and de Sitter cosmological horizon [14] were studied. Zhang and Zhao extended this method from spherical state to the general axisymmetric Kerr and Kerr-Newman black holes, and even investigated more general massive and charged tunneling [15, 16, 17, 18]. More recently, Yang et al. [19] studied 
the Hawking radiation as tunneling from stationary axisymmetric Kerr-Newman-de Sitter black holes. They all got a satisfying result. In addition, there were a lot of works $[20,21,22,23,24,25,26,27,28,29,30,31,32,33$ in the last several years that have attracted the interest of the scientific community. All of these works indicate that the true Hawking radiate spectra are not pure thermal when the self-gravitation is taken into account.

In this paper we apply Kraus-Parikh-Wilzcek's method to investigate the Hawking radiation as tunneling from stationary axisymmetric Kerr-Newman black hole spacetime in the de Sitter universe endowed with NUT (magnetic mass) and magnetic monopole parameters, the metric of which can be written as

$$
\mathrm{d} s^{2}=\frac{\Sigma}{\Delta_{\theta}} \mathrm{d} \theta^{2}+\frac{\Sigma}{\Delta_{r}} \mathrm{~d} r^{2}+\frac{\Delta_{\theta} \sin ^{2} \theta}{\Sigma}\left(a \mathrm{~d} t_{H K}-\frac{\rho}{\Xi} \mathrm{d} \varphi\right)^{2}-\frac{\Delta_{r}}{\Sigma}\left(\mathrm{d} t_{H K}-\frac{\mathcal{A}}{\Xi} \mathrm{d} \varphi\right)^{2},
$$

where

$$
\begin{aligned}
\Sigma & =r^{2}+(n+a \cos \theta)^{2}, \quad \Delta_{\theta}=1+\frac{a^{2}}{\ell^{2}} \cos ^{2} \theta, \quad \ell^{2}=\frac{3}{\Lambda}, \\
\Delta_{r} & =\rho\left[1-\frac{1}{\ell^{2}}\left(r^{2}+5 n^{2}\right)\right]-2\left(M r+n^{2}\right)+q_{e}^{2}+q_{m}^{2}, \\
\rho & =r^{2}+a^{2}+n^{2}, \quad \Xi=1+\frac{a^{2}}{\ell^{2}}, \quad \mathcal{A}=a \sin ^{2} \theta-2 n \cos \theta,
\end{aligned}
$$

$t_{H K}$ being the coordinate time of the spacetime. Beside the cosmological parameter $\Lambda$, the metric (1) possesses five parameters: $M$ the mass parameter, $a$ the angular momentum per unit mass parameter, $n$ the NUT (magnetic mass) parameter, $q_{e}$ the electric charge parameter, and $q_{m}$ the magnetic monopole parameter.

The metric (11) solves the Einstein-Maxwell field equations with an electromagnetic vector potential

$$
A=-\frac{q_{e} r}{\sqrt{\sum \Delta_{r}}} e^{0}-\frac{q_{m} \cos \theta}{\sqrt{\sum \Delta_{\theta}} \sin \theta} e^{3},
$$

and an associated field strength tensor given by

$$
\begin{aligned}
F= & -\frac{1}{\Sigma^{2}}\left[q_{e}\left(r^{2}-a^{2} \cos ^{2} \theta\right)+2 q_{m} r a \cos \theta\right] e^{0} \wedge e^{1} \\
& +\frac{1}{\Sigma^{2}}\left[q_{m}\left(r^{2}-a^{2} \cos ^{2} \theta\right)-2 q_{e} r a \cos \theta\right] e^{2} \wedge e^{3},
\end{aligned}
$$

where we have defined the vierbein field

$$
\begin{aligned}
e^{0} & =\sqrt{\frac{\Delta_{r}}{\Sigma}}\left(\mathrm{d} t_{H K}-\frac{\mathcal{A}}{\Xi} \mathrm{d} \varphi\right), \quad e^{1}=\sqrt{\frac{\Sigma}{\Delta_{r}}} \mathrm{~d} r \\
e^{2} & =\sqrt{\frac{\Sigma}{\Delta_{\theta}}} \mathrm{d} \theta, \quad e^{3}=\sqrt{\frac{\Delta_{\theta}}{\Sigma}} \sin \theta\left(a \mathrm{~d} t_{H K}-\frac{\rho}{\Xi} \mathrm{d} \varphi\right) .
\end{aligned}
$$


We call the spacetime described by the metric (1) a hot NUT-Kerr-Newman-Kasuya (or, for brevity, H-NUT-KN-K) spacetime, since the de Sitter spacetime has been interpreted as being hot [34]. There is a renewed interest in the cosmological parameter as it is found to be present in the inflationary scenario of the early universe. In this scenario the universe undergoes a stage where it is geometrically similar to de Sitter space [35]. Among other things inflation has led to the cold dark matter. If the cold dark matter theory proves correct, it would shed light on the unification of forces ([36], [37]). The monopole hypothesis was propounded by Dirac relatively long ago. The ingenious suggestion by Dirac that magnetic monopole does exist was neglected due to the failure to detect such object. However, in recent years, the development of gauge theories has shed new light on it. Moreover, the H-NUT-KN-K spacetime includes, among others, the physically interesting black hole spacetimes as well as the NUT spacetime which is sometimes considered as unphysical. The curious properties of the NUT spacetime induced Misner [38] to consider it "as a counter example to almost anything". This spacetime plays a significant role in exhibiting the type of effects that can arise in strong gravitational fields.

If we set $\ell \rightarrow \infty, a=q_{e}=q_{m}=0$ in Eq.(1), it then results the NUT metric which is singular along the axis of symmetry $\theta=0$ and $\theta=\pi$. Because of the axial singularities the metric admits different physical interpretations. Misner [39] introduced a periodic time coordinate to remove the singularity, but this makes the metric an uninteresting particlelike solution. To avoid a periodic time coordinate, Bonnor [40] removed the singularity at $\theta=0$ and related the singularity at $\theta=\pi$ to a semiinfinite massless source of angular momentum along the axis of symmetry. This is analogous to representing the magnetic monopole in electromagnetic theory by semiinfinite solenoid [41]. The singularity along $z$-axis is analogous to the Dirac string.

McGuire and Ruffini [42] suggested that the spaces endowed with the NUT parameter should never be directly physically interpreted. To make a physically reasonable solution Ahmed [43] used Bonnor's interpretation of the NUT parameter, i.e., the NUT parameter $n$ is due to the strength of the physical singularity on $\theta=\pi$, and further considered that $n=a$. That means, the angular momentum of the mass $M$ and the angular momentum of massless rod coalesce, and in this case, the metric (1) gives a new black hole solution which poses to solve an outstanding problem of thermodynamics and black hole physics. In view of all the above considerations the work of this paper is interesting.

We organize the paper as follows. In section 2 we derive the horizons and the infinite red-shift surface for the H-NUT-KN-K spacetime. We obtain Hawking thermal spectrum in dragging coordinate system from Klein-Gordon equation in section 3, Although the infinite red-shift surface and the horizons are coincident with each other in the dragging coordinate system, there still exists a coordinate singularity at the horizon of the spacetime and it brings us inconvenience to investigate the tunneling behavior across the 
horizon of the spacetime. In section 4 we remove the coordinate singularity by expressing the metric in the Painlevé coordinate system and then investigate the tunneling radiation characteristics of the H-NUT-KN-K spacetime. Finally, in section 5 we present our concluding remarks.

\section{Horizons and Infinite Red-shift Surface of H-NUT- KN-H Spacetime}

The null surface equation $g^{\mu \nu} \partial_{\mu} f \partial_{\nu} f=0$ gives

$$
r^{4}+\left(a^{2}+6 n^{2}-\ell^{2}\right) r^{2}+2 M \ell^{2} r-\left\{\left(a^{2}-n^{2}+q_{e}^{2}+q_{m}^{2}\right) \ell^{2}-5 n^{2}\left(a^{2}+n^{2}\right)\right\}=0 .
$$

Its roots locate the horizons of the H-NUT-KN-K spacetime. Let us write it in the form

$$
x^{4}+\alpha x^{2}+\beta x+\gamma=0
$$

Under the conditions

$$
\begin{gathered}
\alpha<0, \quad \beta>0, \quad \gamma<0, \quad \alpha^{2}+12 \gamma>0 \\
\left(\alpha^{2}+12 \gamma\right)^{3}>\left(\alpha^{3}-36 \alpha \gamma+\frac{27}{2} \beta^{2}\right)^{2}
\end{gathered}
$$

equation (77) has four real roots: three of which are positive $x_{1}^{+}, x_{2}^{+}, x_{3}^{+}$, and one which is negative $x^{-}$,

$$
\begin{aligned}
& x_{1}^{+}+x_{2}^{+}+x_{3}^{+}+x^{-}=0 \\
& x_{1}^{+}=-w_{1}+w_{2}+w_{3}, \\
& x_{2}^{+}=w_{1}-w_{2}+w_{3}, \\
& x_{3}^{+}=w_{1}+w_{2}-w_{3},
\end{aligned}
$$

where

$$
\begin{aligned}
& w_{1}=\left[-\frac{1}{6} \alpha+\frac{1}{6} \sqrt{\left(\alpha^{2}+12 \gamma\right)} \cos \left(\frac{1}{3} \psi\right)\right]^{1 / 2}, \\
& w_{2}=\left[-\frac{1}{6} \alpha-\frac{1}{6} \sqrt{\left(\alpha^{2}+12 \gamma\right)} \cos \left(\frac{1}{3} \psi+\frac{1}{3} \pi\right)\right]^{1 / 2}, \\
& w_{3}=\left[-\frac{1}{6} \alpha-\frac{1}{6} \sqrt{\left(\alpha^{2}+12 \gamma\right)} \cos \left(\frac{1}{3} \psi-\frac{1}{3} \pi\right)\right]^{1 / 2}, \\
& \cos \psi=\frac{\alpha^{3}-36 \alpha \gamma+\frac{27}{2} \beta^{2}}{\left(\alpha^{2}+12 \gamma\right)^{3 / 2}} .
\end{aligned}
$$

All the four roots of (6) can be evaluated according to the formulae (9)-(12). We denote the negative root by $r_{-}$, and the remaining three positive real roots which represent the horizons of the H-NUT-KN-K spacetime, namely, the inner, outer (event), and 
cosmological horizon, respectively, are given by

$$
\begin{aligned}
& r_{0}=-t_{1}+t_{2}+t_{3}, \\
& r_{H}=t_{1}-t_{2}+t_{3}, \\
& r_{C}=t_{1}+t_{2}-t_{3},
\end{aligned}
$$

where

$$
\begin{aligned}
& t_{1}=\left[\frac{1}{6}\left(\ell^{2}-6 n^{2}-a^{2}\right)+\frac{1}{6} \sqrt{\left\{\left(\ell^{2}-6 n^{2}-a^{2}\right)^{2}-\digamma\right\}} \cos \left(\frac{1}{3} \psi\right)\right]^{1 / 2}, \\
& t_{2}=\left\{\frac{1}{6}\left(\ell^{2}-6 n^{2}-a^{2}\right)-\frac{1}{6} \sqrt{\left\{\left(\ell^{2}-6 n^{2}-a^{2}\right)^{2}-\digamma\right\}} \cos \left(\frac{1}{3} \psi+\frac{1}{3} \pi\right)\right\}^{1 / 2}, \\
& t_{3}=\left\{\frac{1}{6}\left(\ell^{2}-6 n^{2}-a^{2}\right)-\frac{1}{6} \sqrt{\left\{\left(\ell^{2}-6 n^{2}-a^{2}\right)^{2}-\digamma\right\}} \cos \left(\frac{1}{3} \psi-\frac{1}{3} \pi\right)\right\}^{1 / 2}, \\
& \cos \psi=-\frac{\left(\ell^{2}-6 n^{2}-a^{2}\right)\left\{\left(\ell^{2}-6 n^{2}-a^{2}\right)^{2}+3 \digamma\right\}-54 M^{2} \ell^{4}}{\left\{\left(\ell^{2}-6 n^{2}-a^{2}\right)^{2}-\digamma\right\}^{3 / 2}}, \\
& \digamma=12\left\{\left(q_{e}^{2}+q_{m}^{2}-n^{2}+a^{2}\right) \ell^{2}-5 n^{2}\left(a^{2}+n^{2}\right)\right\},
\end{aligned}
$$

under the conditions

$$
\begin{aligned}
\left\{\left(\ell^{2}-6 n^{2}-a^{2}\right)^{2}-\digamma\right\}^{3} & >\left\{\left(\ell^{2}-6 n^{2}-a^{2}\right)^{3}+3\left(\ell^{2}-6 n^{2}-a^{2}\right) \digamma-54 M^{2} \ell^{4}\right\}^{2} \\
\left(\ell^{2}-6 n^{2}-a^{2}\right) & >0 .
\end{aligned}
$$

For the constant time-slice and $r=r_{H}$, the metric (10) reduces to

$$
\mathrm{d} \sigma^{2}=\frac{\Sigma}{\Delta_{\theta}} \mathrm{d} \theta^{2}+\frac{\Delta_{\theta} \sin ^{2} \theta}{\Xi^{2} \Sigma}\left(r_{H}^{2}+a^{2}+n^{2}\right)^{2} \mathrm{~d} \varphi^{2},
$$

the determinant of this two-dimensional line element is

$$
g=\frac{\sin ^{2} \theta}{\Xi^{2}}\left(r_{H}^{2}+a^{2}+n^{2}\right)^{2}
$$

Then the area of the event horizon can be expressed as

$$
A_{H}=\int \mathrm{d} A^{\prime}=\int \sqrt{g} \mathrm{~d} \theta \mathrm{d} \varphi=\frac{4 \pi}{\Xi}\left(r_{H}^{2}+a^{2}+n^{2}\right),
$$

and that of the cosmological horizon as

$$
A_{C}=\frac{4 \pi}{\Xi}\left(r_{C}^{2}+a^{2}+n^{2}\right) .
$$

For the infinite red-shift surface: $g_{00}=0$, we obtain

$$
\Delta_{r}-\Delta_{\theta} a^{2} \sin ^{2} \theta=0 .
$$


It is obvious that the infinite red-shift surface and the event horizon of the spacetime are not coincident with each other, an energy layer exists between them. So the geometrical opticts limit cannot be used here. We therefore carry on dragging coordinate transformation and let

$$
\dot{\varphi}=\frac{\mathrm{d} \varphi}{\mathrm{d} t_{H K}}=-\frac{g_{03}}{g_{33}}=\Omega .
$$

The spacetime line element (11) in the dragging coordinate system is

$$
\mathrm{d} s^{2}=\hat{g}_{00} \mathrm{~d} t_{H K}^{2}+\frac{\Sigma}{\Delta_{r}} \mathrm{~d} r^{2}+\frac{\Sigma}{\Delta_{\theta}} \mathrm{d} \theta^{2}
$$

where

$$
\hat{g}_{00}=g_{00}-\frac{g_{03}^{2}}{g_{33}}=-\frac{\Delta_{\theta} \Delta_{r}(\rho-a \mathcal{A})^{2} \sin ^{2} \theta}{\Sigma\left(\Delta_{\theta} \rho^{2} \sin ^{2} \theta-\Delta_{r} \mathcal{A}^{2}\right)} .
$$

In fact, the line element (23) represents a three-dimensional hypersurface in the fourdimensional H-NUT-KN-K spacetime. Evidently, the infinite red-shift surface is coincident with the horizons of the spacetime in the dragging coordinate system when $\hat{g}_{00}$ vanishes.

\section{Hawking Thermal Spectrum of H-NUT-KN-K Space- time in Dragging Coordinate System}

In this section, for the sake of simplicity, we investigate the Hawking thermal radiation spectrum of uncharged particles. The Klein-Gordon equation for uncharged particles in the curved spacetime can be expressed in the form

$$
\frac{1}{\sqrt{-g}} \frac{\partial}{\partial x^{\mu}}\left(\sqrt{-g} g^{\mu \nu} \frac{\partial}{\partial x^{\nu}} \Phi\right)=\mu^{2} \Phi
$$

With

$$
\Phi=e^{-\mathrm{i} \omega t_{H K}} R(r) \Theta(\theta) e^{\mathrm{i} m \varphi},
$$

$g^{\mu \nu}$ from Eq.(23), and considering the dragging coordinate transformation (22), we obtain the following expression

$$
\begin{aligned}
\frac{\mathrm{d}^{2} R(r)}{\mathrm{d} r^{2}} & +\frac{1}{g^{11}}\left(\frac{g^{11}}{\sqrt{-g}} \frac{\partial}{\partial r} \sqrt{-g}+\frac{\partial g^{11}}{\partial r}\right) \frac{\mathrm{d} R(r)}{\mathrm{d} r}+\frac{1}{g^{11}} \frac{R(r)}{\Theta(\theta)}\{G(r, \theta)\} \\
& =\frac{1}{g^{11}}\left[\mu^{2}+\left(\omega+m \frac{g_{03}}{g_{33}}\right)^{2} \hat{g}^{00}\right] R(r),
\end{aligned}
$$

where

$$
G(r, \theta)=\frac{\Delta_{\theta}}{\Sigma} \frac{\mathrm{d}^{2} \Theta(\theta)}{\mathrm{d} \theta^{2}}+\frac{1}{\sqrt{-g}} \frac{\partial}{\partial \theta}\left(\sqrt{-g} g^{22}\right) \frac{\mathrm{d} \Theta(\theta)}{\mathrm{d} \theta}
$$


Introducing the tortoise coordinate

$$
r_{*}=\frac{1}{2 \kappa_{H}} \ln \left(r-r_{H}\right)
$$

we have

$$
\begin{aligned}
\frac{\mathrm{d}^{2} R(r)}{\mathrm{d} r_{*}^{2}} & -2 \kappa_{H} \frac{\mathrm{d} R(r)}{\mathrm{d} r_{*}}+2 \kappa_{H}\left(r-r_{H}\right)\left(\frac{1}{\sqrt{-g}} \frac{\partial \sqrt{-g}}{\partial r}+\frac{1}{g^{11}} \frac{\partial g^{11}}{\partial r}\right) \frac{\mathrm{d} R(r)}{\mathrm{d} r_{*}} \\
& +\frac{4 \kappa_{H}^{2}\left(r-r_{H}\right)^{2}}{g^{11}} \frac{R(r)}{\Theta(\theta)}\{G(r, \theta)\} \\
& =\frac{4 \kappa_{H}^{2}\left(r-r_{H}\right)^{2}}{g^{11}}\left[\mu^{2}+\left(\omega+m \frac{g_{03}}{g_{33}}\right)^{2} \hat{g}^{00}\right] R(r),
\end{aligned}
$$

where

$$
\kappa_{H}=\frac{1}{2 \ell^{2}\left(r_{H}^{2}+a^{2}+n^{2}\right)}\left(r_{H}-r_{-}\right)\left(r_{H}-r_{0}\right)\left(r_{C}-r_{H}\right)
$$

is the surface gravity of the event horizon. In the vicinity of the event horizon, i.e., when $r \rightarrow r_{H}$, one could find that

$$
\frac{4 \kappa_{H}^{2}\left(r-r_{H}\right)^{2}}{g^{11}}\left[\mu^{2}+\left(\omega+m \frac{g_{03}}{g_{33}}\right)^{2} \hat{g}^{00}\right] R(r) \equiv-\left(\omega-m \Omega_{H}\right)^{2} R(r) .
$$

Equation (30) then can be put, near the horizon, in the standard wave equation form:

$$
\frac{\mathrm{d}^{2} R(r)}{\mathrm{d} r_{*}^{2}}+\left(\omega-\omega_{0}\right)^{2} R(r)=0
$$

where $\omega_{0}=m \Omega_{H}=\frac{\Xi a m}{r_{H}^{2}+a^{2}+n^{2}}, \Omega_{H}$ being the dragging angular velocity at the event horizon. Solving Eq.(33) we obtain the ingoing and outgoing radial wave functions for uncharged particles in the H-NUT-KN-K spacetime as follows:

$$
\Phi_{\text {in }}=e^{-\mathrm{i} \omega v}, \quad \Phi_{\text {out }}=e^{-\mathrm{i} \omega v} e^{2 \mathrm{i}\left(\omega-\omega_{0}\right) r_{*}}
$$

where $v=t_{H K}+\frac{\omega-\omega_{0}}{\omega} r_{*}$ is the advanced Eddington-Finkelstein coordinate. Near the event horizon, $\Phi_{\text {out }}$ can be written as

$$
\Phi_{\text {out }}=e^{-\mathrm{i} \omega v}\left(r-r_{H}\right)^{\mathrm{i}\left(\omega-\omega_{0}\right) / \kappa_{H}}
$$

The $\Phi_{\text {out }}$ has a logarithm singularity. By analytical continuation rotating $-\pi$ through the lower-half complex $r$-plane, we have

$$
\left(r-r_{H}\right) \rightarrow\left|r-r_{H}\right| e^{-\mathrm{i} \pi}=\left(r-r_{H}\right) e^{-\mathrm{i} \pi}
$$

Damour and Ruffini's work [44] of generalizing the classical approach of barrier penetration to curved spaces endowed with future horizons, allows one to recover most directly 
the spectrum of the Hawking radiation. The existence of a spacelike Killing vector $\xi_{t}$ inside the horizon permits a classical particle as "seen" from infinity to reach a negativeenergy state. In the quantum description, this phenomenon allows an antiparticle to reach positive-energy states, and these states can be tunneled out by a wave function "over" the horizon. This gives rise to the creation of a pair: one particle (positive energy) going out and one antiparticle (negative energy) falling back toward the singularity.

Using the Damour-Ruffini stretch method of analysis, and extending it to the inside of the event horizon, we obtain the spectrum of the Hawking radiation [44]

$$
N_{\omega}=\frac{1}{e^{\left(\omega-\omega_{0}\right) / T}-1}=\frac{1}{e^{\alpha_{H} A_{H}}-1},
$$

where

$$
T=\frac{\kappa_{H}}{2 \pi}, \quad \alpha_{H}=\frac{3\left(\omega-\omega_{0}\right) \Xi \ell^{2}}{\left[-\digamma r_{H}^{-1}+18 M \ell^{2}+6\left(a^{2}+6 n^{2}-\ell^{2}\right) r_{H}\right]},
$$

$A_{H}$ being the area of the event horizon and $\digamma$ is given by (15). It is obvious from Eq.(37) that the Hawking radiation spectrum at the event horizon is related to the fixed area of the event horizon.

In similar fashion, the Hawking radiation spectrum at the cosmological horizon is given by

$$
N_{\omega}=\frac{1}{e^{\left(\omega-\omega_{0}\right) / T}-1}=\frac{1}{e^{\alpha_{C} A_{C}}-1}
$$

where

$$
T=\frac{\kappa_{C}}{2 \pi}, \quad \alpha_{C}=\frac{3\left(\omega-\omega_{0}\right) \Xi \ell^{2}}{\left[-\digamma r_{C}^{-1}+18 M \ell^{2}+6\left(a^{2}+6 n^{2}-\ell^{2}\right) r_{C}\right]},
$$

Equation (39) shows that the derived Hawking radiation spectrum at the cosmological horizon is also related to the fixed area of the cosmological horizon, $A_{C}$.

Thus the thermal property of the H-NUT-KN-K spacetime can be derived in the dragging coordinate system. The expressions (37) and (39) are based on the fixed background spacetime. In fact, the horizons change with the emission, and the background spacetime is dynamical.

\section{Painlevé Coordinate Transformation and Tunnel- ing Process of Uncharged Particles from H-NUT- KN-K Spacetime}

\subsection{Painlevé-H-NUT-KN-K Metric}

In the dragging coordinate system, the infinite red-shift surface coincides with the horizons, but still there is a coordinate singularity at the horizon of the spacetime, which brings us inconvenience to investigate the tunneling process across the horizon. So we 
perform general Painlevé coordinate transformation [45] to eliminate the coordinate singularity from the metric (23) as follows. We write

$$
\mathrm{d} t_{H K}=\mathrm{d} t+F(r, \theta) \mathrm{d} r+G(r, \theta) \mathrm{d} \theta
$$

where $F(r, \theta)$ and $G(r, \theta)$ are two functions to be determined about $r, \theta$, and satisfy the integrability condition $\partial_{\theta} F(r, \theta)=\partial_{r} G(r, \theta)$. Substituting Eq.(41) into the metric (23), and ordering the derived constant-time slice of the spacetime flat Euclidean in the radial, we obtain the metric in the general Painlevé coordinate system:

$$
\begin{aligned}
\mathrm{d} s^{2}= & \hat{g}_{00} \mathrm{~d} t^{2}+\mathrm{d} r^{2} \pm 2 \sqrt{\hat{g}_{00}\left(1-g_{11}\right)} \mathrm{d} t \mathrm{~d} r+\left[\hat{g}_{00} G^{2}(r, \theta)+g_{22}\right] \mathrm{d} \theta^{2} \\
& +2 \sqrt{\hat{g}_{00}\left(1-g_{11}\right)} G(r, \theta) \mathrm{d} r \mathrm{~d} \theta+2 \hat{g}_{00} G(r, \theta) \mathrm{d} t \mathrm{~d} \theta
\end{aligned}
$$

where the positive sign $(+)$ represents the spacetime of the outgoing particle, and the negative sign (-) denotes the metric of the ingoing particle. Flat Euclidean space consideration gives

$$
F(r, \theta)= \pm \sqrt{\left(1-g_{11}\right) / \hat{g}_{00}}
$$

According to Landau's condition of coordinate clock synchronization [46]

$$
\frac{\partial}{\partial x^{i}}\left(-\frac{g_{0 j}}{\hat{g}_{00}}\right)=\frac{\partial}{\partial x^{j}}\left(-\frac{g_{0 i}}{\hat{g}_{00}}\right)
$$

from which, for the metric (42), we also have

$$
\frac{\partial F(r, \theta)}{\partial \theta}=\frac{\partial G(r, \theta)}{\partial r}
$$

Thus the Painlevé-H-NUT-KN-K metric (42) satisfies the Landau's condition of the coordinate clock synchronization. In addition, there are many other superior features: firstly, the metric is regular at the horizons; secondly, the infinite red-shift surface and the horizons are coincident with each other; thirdly, spacetime is stationary; and fourthly, constant-time slices are just flat Euclidean space in radial. All of these properties are advantageous for us to study the Hawking thermal spectrum via tunneling.

In order to investigate the tunneling behavior of the uncharged particles from the horizon, we first evaluate the radial, null geodesics. Since the tunneling processes take place near the event horizon, we may consider a particle tunneling across the event horizon as an ellipsoid shell and think that the particle should still be an ellipsoid shell during the tunneling process, i.e., the particle does not have motion in the $\theta$-direction. Under these assumptions $(\mathrm{d} s=0=\mathrm{d} \theta)$, the radial, null geodesics followed by uncharged particles are, from (42),

$$
\dot{r}=\frac{d r}{d t}=\frac{\sqrt{\Delta_{\theta}}(\rho-a \mathcal{A}) \sin \theta\left( \pm \sqrt{\Sigma}-\sqrt{\Sigma-\Delta_{r}}\right)}{\sqrt{\Sigma\left(\Delta_{\theta} \rho^{2} \sin ^{2} \theta-\Delta_{r} \mathcal{A}^{2}\right)}},
$$

where the plus (minus) sign indicates the outgoing (ingoing) geodesics, under the implicit assumption that time $t$ increases towards the future. 


\subsection{Tunneling Process}

We now turn to discuss the Hawking radiation of uncharged particles as a semi-classical tunneling process across the barrier which is created just by the outgoing particle itself. We adopt the picture of a pair of virtual particles spontaneously created just inside the horizon. The positive energy virtual particle can tunnel out and materialize as a real particle escaping classically to infinity, and the negative energy anti-particle is absorbed by the H-NUT-KN-K spacetime, resulting in a decrease in the mass and angular momentum of the H-NUT-KN-K spacetime. We consider the particle as an ellipsoid shell of energy $\omega$ and angular momentum $a \omega$. If the particle's self-gravitation is taken into account, Eqs.(13), (42), and (46) should be modified. To guarantee the conservation of energy and angular momentum, we fix the total mass and total angular momentum of the whole of the spacetime (i.e., the H-NUT-KN-K spacetime plus the outside spacetime) but allow the the H-NUT-KN-K spacetime's mass and angular momentum to fluctuate. When the particle is tunneled out as an ellipsoid shell of energy $\omega$ and angular momentum $a \omega$, then the mass and angular momentum of the H-NUT-KN-K spacetime will be replaced by $(M-\omega)$ and $a(M-\omega)$, respectively. Meanwhile, the event horizon will shrink, we refer to the cases pre- and post-shrinking as two turning points of potential barrier. The distance between the two turning points is the width of potential barrier and decided by the energy of outgoing particle. In this critical situation, Eqs.(13), (42), and (46) will be modified by replacing the mass parameter $M$ with $(M-\omega)$, and the shell of energy will move along the modified null geodesic in the radial direction

$$
\dot{r}=\frac{\sqrt{\Delta_{\theta}}(\rho-a \mathcal{A}) \sin \theta\left( \pm \sqrt{\Sigma}-\sqrt{\Sigma-\Delta_{r}^{\prime}}\right)}{\sqrt{\Sigma\left(\Delta_{\theta} \rho^{2} \sin ^{2} \theta-\Delta_{r}^{\prime} \mathcal{A}^{2}\right)}}
$$

where

$$
\Delta_{r}^{\prime}=\left(r^{2}+a^{2}+n^{2}\right)\left[1-\frac{1}{\ell^{2}}\left(r^{2}+5 n^{2}\right)\right]-2\left\{(M-\omega) r+n^{2}\right\}+q_{e}^{2}+q_{m}^{2}
$$

is the horizon equation after the emission of the particle with energy $\omega$.

In the WKB approximation, the tunneling probability for an outgoing positive energy particle can be expressed in terms of the imaginary part of the action as

$$
\Gamma \sim e^{-2 \operatorname{Im} S}
$$

At this point, it should be noticed that the coordinate $\varphi$ does not appear in the metric in the dragging coordinate system. That is, $\varphi$ is an ignorable coordinate in the Lagrangian function, $L(r, \dot{r}, \varphi, \dot{\varphi}, t)$. To eliminate this degree of freedom completely, the imaginary 
part of the action should be written as

$$
\begin{aligned}
\operatorname{Im} S & =\operatorname{Im} \int_{t_{i}}^{t_{f}}\left(p_{r} \dot{r}-p_{\varphi} \dot{\varphi}\right) \mathrm{d} t \\
& =\operatorname{Im} \int_{r_{i}}^{r_{f}}\left[\int_{(0,0)}^{\left(p_{r}, p_{\varphi}\right)}\left(\dot{r} \mathrm{~d} p_{r}^{\prime}-\dot{\varphi} \mathrm{d} p_{\varphi}^{\prime}\right)\right] \frac{\mathrm{d} r}{\dot{r}},
\end{aligned}
$$

where $p_{r}$ and $p_{\varphi}$ are two canonical momenta conjugate to $r$ and $\varphi$, respectively. The $r_{i}$ and $r_{f}$ are just inside and outside the barrier at the event horizon through which the particle tunnels.

We now remove the momentum in favor of energy by applying the Hamilton's equations

$$
\begin{aligned}
\dot{r} & =\left.\frac{\mathrm{d} H}{\mathrm{~d} p_{r}}\right|_{\left(r ; \varphi, p_{\varphi}\right)}, \\
\dot{\varphi} & =\left.\frac{\mathrm{d} H}{\mathrm{~d} p_{\varphi}}\right|_{\left(\varphi ; r, p_{r}\right)},\left.\quad \mathrm{d} H\right|_{\left(\varphi ; r, p_{r}\right)}=\Omega^{\prime} \mathrm{d} J,
\end{aligned}
$$

where $H=\frac{M}{\Xi^{2}}$ is the total energy of the H-NUT-KN-K spacetime, and when the particle of energy $\omega$ is propagating from inside to outside the event horizon, then $H=\frac{M-\omega}{\Xi^{2}}$, $\mathrm{d} H=-\frac{1}{\Xi^{2}} \mathrm{~d} \omega, p_{\varphi}=J$.

Substituting Eqs.(47) and (50) into Eq.(49), we obtain

$$
\begin{aligned}
\operatorname{Im} S= & \operatorname{Im} \int_{\frac{M}{\Xi^{2}}}^{\frac{M-\omega}{\Xi^{2}}} \int_{r_{i}}^{r_{f}}\left(\frac{\mathrm{d} H^{\prime}}{\dot{r}}-\frac{\Omega^{\prime} \mathrm{d} J^{\prime}}{\dot{r}}\right) \mathrm{d} r=\operatorname{Im} \int_{0}^{\omega} \int_{r_{i}}^{r_{f}}-\frac{1}{\Xi^{2}}\left(\frac{\mathrm{d} \omega^{\prime}}{\dot{r}}-\frac{a \Omega^{\prime} \mathrm{d} \omega^{\prime}}{\dot{r}}\right) \mathrm{d} r \\
= & \operatorname{Im} \int_{0}^{\omega} \int_{r_{i}}^{r_{f}}-\frac{1}{\Xi^{2}} \frac{\sqrt{\left(\Delta_{\theta} \rho^{2} \sin ^{2} \theta-\tilde{\Delta}_{r}^{\prime} \mathcal{A}^{2}\right)}\left[\Sigma \sqrt{\Delta_{\theta}}+\sqrt{\Sigma \Delta_{\theta}\left(\Sigma-\tilde{\Delta}_{r}^{\prime}\right)}\right]}{\Delta_{\theta}(\rho-a \mathcal{A}) \sin \theta \tilde{\Delta}_{r}^{\prime}} \\
& \times\left(1-a \Omega^{\prime}\right) \mathrm{d} \omega^{\prime} \mathrm{d} r
\end{aligned}
$$

where

$$
\tilde{\Delta}_{r}^{\prime}=\left(r^{2}+a^{2}+n^{2}\right)\left[1-\frac{1}{\ell^{2}}\left(r^{2}+5 n^{2}\right)\right]-2\left\{\left(M-\omega^{\prime}\right) r+n^{2}\right\}+q_{e}^{2}+q_{m}^{2} .
$$

There is a single pole in Eq.(51) at the event horizon of the H-NUT-KN-K spacetime after the particle emission. We can evaluate the integral by deforming the contour around the pole, so as to ensure that positive energy solution decay in time. In this way, we finish firstly the $\omega^{\prime}$ integral and obtain the result

$$
\operatorname{Im} S=\operatorname{Im} \int_{r_{i}}^{r_{f}}-\frac{\pi r \mathrm{i}}{\Xi} \mathrm{d} r=-\frac{\pi}{2 \Xi}\left(r_{f}^{2}-r_{i}^{2}\right) .
$$

In terms of the entropy expression $S_{B H}=\pi\left(r_{H}^{2}+a^{2}+n^{2}\right) / \Xi$, the tunneling rate at the event horizon can then be expressed, using Eq.(48), as follows:

$$
\Gamma \sim e^{-2 \operatorname{Im} S}=e^{\Delta S_{B H}}
$$


where $\Delta S_{B H}=S_{B H}^{\prime}-S_{B H}$ is the difference of Bekenstein-Hawking entropies of the HNUT-KN-K spacetime before and after the emission of the particle. From comparison of Eqs.(153) and (37), we can learn that the tunneling rate at the event horizon provides a correct modification to Hawking radiation spectrum.

Let us now discuss the the Hawking radiation of the particle via tunneling at the cosmological horizon. The particle is found tunneled into the cosmological horizon differently from the particle's tunneling behavior of the event horizon. When the particle with energy $\omega$ tunnels into the cosmological horizon, Eqs.(13), (42) and (46) should have to modify by replacing the mass parameter $M$ with $(M+\omega)$ after taking the self-gravitation action into account. Thus, after tunneling the particle with energy $\omega$ into the cosmological horizon, the null radial geodesic takes the form

$$
\dot{r}=\frac{\sqrt{\Delta_{\theta}}(\rho-a \mathcal{A}) \sin \theta\left( \pm \sqrt{\Sigma}-\sqrt{\Sigma-\Delta_{r}^{\prime \prime}}\right)}{\sqrt{\Sigma\left(\Delta_{\theta} \rho^{2} \sin ^{2} \theta-\Delta_{r}^{\prime \prime} \mathcal{A}^{2}\right)}}
$$

where

$$
\Delta_{r}^{\prime \prime}=\left(r^{2}+a^{2}+n^{2}\right)\left[1-\frac{1}{\ell^{2}}\left(r^{2}+5 n^{2}\right)\right]-2\left\{(M+\omega) r+n^{2}\right\}+q_{e}^{2}+q_{m}^{2} .
$$

Different from the event horizon, $H=-\frac{M}{\Xi^{2}}$ and $H^{\prime}=-\frac{M+\omega}{\Xi^{2}}$ are the total energy of the H-NUT-KN-K spacetime before and after the particle with energy $\omega$ tunnels into. Then the imaginary part of the action at the cosmological horizon can be written as

$$
\begin{aligned}
\operatorname{Im} S= & \operatorname{Im} \int_{-\frac{M}{\Xi^{2}}}^{-\frac{M-\omega}{\Xi^{2}}} \int_{r_{C i}}^{r_{C f}}\left(\frac{\mathrm{d} H^{\prime}}{\dot{r}}-\frac{\Omega^{\prime} \mathrm{d} J^{\prime}}{\dot{r}}\right) \mathrm{d} r=\operatorname{Im} \int_{0}^{\omega} \int_{r_{C i}}^{r_{C f}}-\frac{1}{\Xi^{2}}\left(\frac{\mathrm{d} \omega^{\prime}}{\dot{r}}-\frac{a \Omega^{\prime} \mathrm{d} \omega^{\prime}}{\dot{r}}\right) \mathrm{d} r \\
= & \operatorname{Im} \int_{0}^{\omega} \int_{r_{C i}}^{r_{C f}}-\frac{1}{\Xi^{2}} \frac{\sqrt{\left(\Delta_{\theta} \rho^{2} \sin ^{2} \theta-\tilde{\Delta}_{r}^{\prime \prime} \mathcal{A}^{2}\right)}\left[\Sigma \sqrt{\Delta_{\theta}}+\sqrt{\Sigma \Delta_{\theta}\left(\Sigma-\tilde{\Delta}_{r}^{\prime \prime}\right)}\right]}{\Delta_{\theta}(\rho-a \mathcal{A}) \sin \theta \tilde{\Delta}_{r}^{\prime \prime}} \\
& \times\left(1-a \Omega^{\prime}\right) \mathrm{d} \omega^{\prime} \mathrm{d} r,
\end{aligned}
$$

where

$$
\tilde{\Delta}_{r}^{\prime \prime}=\left(r^{2}+a^{2}+n^{2}\right)\left[1-\frac{1}{\ell^{2}}\left(r^{2}+5 n^{2}\right)\right]-2\left\{\left(M+\omega^{\prime}\right) r+n^{2}\right\}+q_{e}^{2}+q_{m}^{2},
$$

$r_{C i}$ and $r_{C f}$ are the locations of the cosmological horizon before and after the particle of energy $\omega$ tunneling into. There exists a single pole at the cosmological horizon in Eq.(155). Finishing the $\omega^{\prime}$ integral firstly, we obtain

$$
\operatorname{Im} S=\operatorname{Im} \int_{r_{C i}}^{r_{C f}}-\frac{\pi r \mathrm{i}}{\Xi} \mathrm{d} r=-\frac{\pi}{2 \Xi}\left(r_{C f}^{2}-r_{C i}^{2}\right) .
$$

Using the entropy expression $S_{C H}=\pi\left(r_{C}^{2}+a^{2}+n^{2}\right) / \Xi$, the tunneling rate at the cosmological horizon is given by

$$
\Gamma \sim e^{-2 \operatorname{Im} S}=e^{\Delta S_{C H}},
$$


where $\Delta S_{C H}=S_{C H}^{\prime}-S_{C H}$ is the difference of Bekenstein-Hawking entropies of the cosmological horizon before and after the particle of energy $\omega$ is tunneling into. Comparing Eqs.(57) and (39), we can find that the tunneling rate at the cosmological horizon still provides a correct modification to Hawking radiation spectrum.

\section{Concluding Remarks}

The main concern of this paper has been exclusively the investigation of the tunneling radiation characteristics of uncharged particles from a more general spacetime, namely, the hot NUT-Kerr-Newman-Kasuya spacetime, by applying Kraus-Parikh-Wilczek's semiclassical quantum tunneling method ([7], 8], [12]). Our result is satisfactory.

In our study, we find that the tunneling rate at the event/cosmological horizon is related to the change of Bekenstein-Hawking entropy and the radiant spectrum is no longer thermal after considering the H-NUT-KN-K spacetime background as dynamical and incorporating the self-gravitation effect of the emitted particles when the energy conservation and angular momentum conservation are taken into account. Thus our study is perfectly extending the Kraus-Parikh-Wilczek's semi-classical tunneling framework in a more general spacetime, containing six parameters: the mass $M$, angular momentum per unit mass $a$, cosmological parameter $\Lambda$, NUT (magnetic mass) parameter $n$, electric charge $q_{e}$ and magnetic monopole charge $q_{m}$.

In special cases, our result reduces to the Reissner-Norström black hole case for $\ell \rightarrow \infty$, $a=0=n$, and to the Schwarzschild black hole case for $\ell \rightarrow \infty, a=0=n, q_{e}=0=q_{m}$, and supports the Parikh's result ([7], [8], [12]).

For $n=0$ and $q_{e}^{2}+q_{m}^{2}=q^{2}$, our study gives the result of Yang et al. [19] for the Kerr-Newman-de Sitter black hole. Indeed, by suitably choosing the parameters of the spacetime, the result of this paper can be specialized for all the interesting black hole spacetimes, de Sitter spacetimes as well as the NUT spacetime which has curious properties as discussed in the introduction. Following Ahmed [43], if one considers the Bonnor's interpretation: the NUT parameter $n$ is due to the strength of the physical singularity on $\theta=\pi$, and further chooses $n=a$, then our study gives result for the interesting coalescing black hole spacetime in the de Sitter universe. In addition, our result can be directly extended to the anti-de Sitter case by changing the sign of the cosmological parameter $\ell^{2}$ to a negative one. In view of all of the above attractive features, the study of this paper is interesting.

Using Kraus-Parikh-Wilzcek's method, Hawking radiation of charged massive particles as a semi-classical tunneling process across the horizons of the H-NUT-KN-K spacetime [47] is interesting as well.

Hawking radiation from the event horizon of black hole is one of the most important 
achievements of quantum field theory in curved spacetimes. In fact, due to Hawking evaporation classical general relativity, statistical physics, and quantum field theory are connected in quantum black hole physics. It is, therefore, generally believed that the deep investigation of black hole physics would be helpful to set up a satisfactory quantum theory of gravity.

\section{Acknowledgement}

I am thankful to SIDA as well as the Abdus Salam International Centre for Theoretical Physics, Trieste, Italy, where this paper was produced during my Associateship visit. 


\section{References}

[1] Hawking, S. W. (1974). Nature 248, 30.

[2] Hawking, S. W. (1975). Communications in Mathematical Physics 43, 199.

[3] Gibbons, G. W., and Hawking, S. W. (1977) Physical Review D 15, 2752.

[4] Hawking, S. W. (1976). Physical Review D 14, 2460.

[5] Hawking, S. W. (2005). Physical Review D 72, 084013.

[6] Parikh, M. K. (2004). General Relativity and Gravitation 36, 2419; hep-th/0402166.

[7] Parikh, M. K. (2004). International Journal of Modern Physics D 13, 2351;

hep-th/0405160.

[8] Parikh, M. K., and Wilczek, F. (2000). Physical Review Letters 85, 5042; hep-th/9907001.

[9] Kraus, P., and Wilczek, F. (1995). Nuclear Physics B 433, 403.

[10] Kraus, P., and Wilczek, F. (1995). Nuclear Physics B 437, 231.

[11] Keski-Vakkuri, E., and Kraus, P. (1996). Physical Review D 54, 7407.

[12] Parikh, M. K. (2002). Physics Letters B 546, 189.

[13] Hemming, S., and Keski-Vakkuri, E. (2001), Physical Review D 64, 44006.

[14] Medved, A. J. M. (2002). in Physical Review D 66, 124009.

[15] Zhang, J., and Zhao, Z. (2005). Modern Physics Letters A 20, 1673.

[16] Zhang, J., and Zhao, Z. (2005). Physics Letters B 618, 14.

[17] Zhang, J., and Zhao, Z. (2005). Nuclear Physics B 725, 173.

[18] Zhang, J., and Zhao, Z. (2005). Journal of High Energy Physics 10, 055.

[19] Yang, S. Z., Jiang, Q. Q., and Li, H. L. (2007). International Journal of Theoretical Physics 46, 625.

[20] Wu, S, Q., and Jiang, Q. Q. (in press). Hawking radiation via tunneling from higher dimensional Reissner-Nordström-de Sitter black hole. hep-th/0603082.

[21] Wu, S. Q., and Jiang, Q. Q. (2006). JHEP 03, 079; hep-th/0602033. 
[22] Jiang, Q. Q., Wu, S. Q., and Cai, X. (2006). Physical Review D 73, 064003, 069902(E); hep-th/0512351.

[23] Jiang, Q. Q., and Wu, S. Q. (2006). Physics Letters B 635, 151; hep-th/0511123.

[24] Arzano, M., Medved, A. J. M., and Vagenas, E. C. (2005). Journal of High Energy Physics 09, 037; hep-th/0505266.

[25] Medved, A. J. M., and Vagenas, E. C. (2005). Modern Physics Letters A 20, 1723; gr-qc/0505015.

[26] Medved, A. J. M., and Vagenas, E. C. (2005). Modern Physics Letters A 20, 2449; gr-qc/0504113.

[27] Angheben, M., Nadalini, M., Vanzo, L., and Zerbini, S. (2005). Journal of High Energy Physics 0505, 014; hep-th/0503081.

[28] Setare, M. R., and Vagenas, E. C. (2005). International Journal of Modern Physics A 20, 7219; hep-th/0405186.

[29] Setare, M. R., and Vagenas, E. C. (2004). Physics Letters B 584, 127; hep-th/0309092.

[30] Vagenas, E. C. (2002). Physics Letters B 533, 302; hep-th/0109108.

[31] Vagenas, E. C. (2002). Modern Physics Letters A 17, 609; hep-th/0108147.

[32] Vagenas, E. C. (2001). Physics Letters B 503, 399; hep-th/0012134.

[33] Vagenas, E. C. (2003). Physics Letters B 559, 65; hep-th/0209185.

[34] Gasperini, M. (1988). Classical and Quantum Gravity 5, 521.

[35] Guth, A. H. (1981). Physical Review D 23, 347.

[36] Turner, M. S. (1995). Fermilab-Conf-95-125A.

[37] Turner, M. S. (1998). Fermilab-Conf-95-126A.

[38] Misner, C. W. (1967). Taub-NUT space as a Counter example to Almost Anything, in Relativity Theory and Astrophysics 1, Lectures in Applied Mathematics, Vol. 8, edited by J. Ehlers, American Mathematical Society, Providence, R. I. p.160.

[39] Misner, C. W. (1963). Journal of Mathematical Physics 4, 924.

[40] Bonnor, W. B. (1969). Proceedings of the Cambridge Philosophical Society 66, 145. 
[41] Dowker, J. S. (1974). General Relativity and Gravitation 5, 603.

[42] Mcguire, P., and Ruffini, R. (1975). Physical Review D 12, 3019.

[43] Ahmed, M. (2007). International Journal of Theoretical Physics 46, 445.

[44] Damour, T., and Ruffini, R. (1976). Physical Review D 14, 332.

[45] Painlevé, P. (1921). Comptes Rendus de l'Academie des Sciences, Serie I (Mathematique) 173, 677.

[46] Landau, L. D., and Lifshitz, E. M. (1987). Classical Theory of Fields, Vol. 2, 4th Version pergamon, New York.

[47] Ali, M. H. (2007). Charged Particles' Tunneling from Hot-NUT-Kerr-NewmanKasuya Spacetime. gr-qc/0707.1079. 